Scientific Visualization, 2020, volume 12, number 3, pages 79 - 88, DOI: 10.26583/sv.12.3.07

\title{
Modeling and visualization of an explosion in sandy soil. Numerical simulation and experiment
}

\author{
V.A. Kuzmin ${ }^{1, A, B}$, V.A. Kikeev²,C,D, S.I. Gerasimov3,A,B,C,D, V.I. Erofeev4,C, V.P. Gandurin5,A, \\ N.A. Trepalov6,A, E.G. Kosyak7,B, P.G. Kuznetsov ${ }^{8, B}$ \\ A Russian Federal Nuclear Center All-Russian Research Institute of Experimental Physics \\ B Sarov State Physics and Technical Institute National Research \\ Nuclear University MEPhI \\ C Institute for Problems of Mechanical Engineering, Institute of Applied Physics, \\ Russian Academy of Sciences \\ D Nizhny Novgorod State Technical University \\ ${ }^{1}$ ORCID: 0000-0002-7141-0408, v a kuzmin@mail.ru \\ 2 ORCID: 0000-0002-2375-0803, vkikeev@mail.ru \\ 3 ORCID: 000o-0002-6850-0816, s.i.gerasimov@mail.ru \\ 4 ORCID: 0000-0002-6637-5564, erfo4@mts-nn.ru \\ 5 ORCID: oooo-0002-8988-1304, v.gandurin@yandex.ru \\ 6 ORCID: oooo-0002-5131-1736, natrepalov@mail.ru \\ 7 ORCID: 0000-0001-6291-2396, keg@sarfti.ru \\ 8 ORCID: 0000-0003-2691-206X, kpg@sarfti.ru
}

\begin{abstract}
The background oriented shlieren (BOS) method is actively used for visualization of flows that occur in a gas environment. Along with it, the capabilities of the method can be used to visualize disturbances in an opaque continuous medium. In this case, in addition to traditional high-speed video shooting, a "background screen" is used in the form of an initial nonuniform randomly distributed picture of the surface grains of the medium being studied. When perturbations are propagated in this area, they are visualized using cross-correlation analysis. It is of interest to determine the sensitivity of the method in the application of important tasks of sensing media by buried blasts. An additional advantage of using the BOS method is its visual verification of the numerical solutions used.

The paper considers an experimental and calculated study of HE blast in a dry sandy ground with a density of $\mathrm{p} \approx 1.5 \mathrm{~g} / \mathrm{cm}^{3}$. During the experiment, optical-physical registration of the process of perturbation development on the ground surface was used. Using a crosscorrelation image processing algorithm, the diameters of the perturbation zone and the height of the dome were determined. The results of numerical simulation are also presented. Comparison of calculated and experimental data on the height of the sandy ground discharge showed their satisfactory correspondence, which indicates the adequacy of the applied mathematical model.
\end{abstract}

Keywords: sandy ground, buried blast, perturbation, ground dome, mathematical model, numerical simulation.

\section{Introduction}

The development of digital systems for image recording and processing has significantly expanded the capabilities of optical diagnostic methods. Among the methods of digital image processing, in the field of optical-physical registration, the cross-correlation algorithm is the most widely used. This algorithm is successfully applied in the shadow background method 
[1] and in the digital tracer visualization method [2]. The use of cross-correlation processing allows us to obtain quantitative information about the studied environment in addition to qualitative data. The experimental data obtained in this way allow to conduct the verification of numerical modeling techniques, which is demonstrated in [3-6].

The purpose of this work is to study the explosion in dry sandy ground, the verification of numerical method, and work out the method of optical and physical registration of the disturbance of the free surface of the ground. The results of the work are planned to be used in the future as the basis for determining the detonation's depth the specified mass of TNT equivalent explosives or mass of TNT explosives at a predetermined depth.

\section{Setting up an experiment}

An experimental study of a buried blast was performed by high-speed video recording of the surface of sandy ground (Figure 1). Two experiments were carried out in which a cylindrical high explosive charge (HE) located at a depth $h$ from the free surface of the field was initiated (0.5 $\mathrm{m}$ in experiment $1,0.5 \mathrm{~m}$ in experiment 2). The mass of $\mathrm{HE}$ in TNT equivalent was 0.113 $\mathrm{kg}$. The initiation of the charge was carried out using a high voltage generator (VG). Simultaneously with the activation of the VG, a high-speed video camera was switched on. Video recording was performed with a frame sequence period of $0.24 \mathrm{~ms}$, a frame exposure time of 50 $\mu \mathrm{s}$, and a resolution of the camera matrix of $1440 \times 700$ pixels. Video recording was performed from a distance of $Z=32 \mathrm{~m}$. The spatial resolution of the video recording scheme in the area of the explosion epicenter was $0.6 \mathrm{pixel} / \mathrm{mm}$.

In experiments, the same pattern of ground dome formation and its expansion was registered. Starting at a time of $\sim 60 \mathrm{~ms}$, the dome begins to fragment. The upward movement of ground particles continues until $\sim 570 \mathrm{~ms}$, then there is a change of direction. Figure 2 shows, as an example, fragments of video registration obtained during experiment 1.

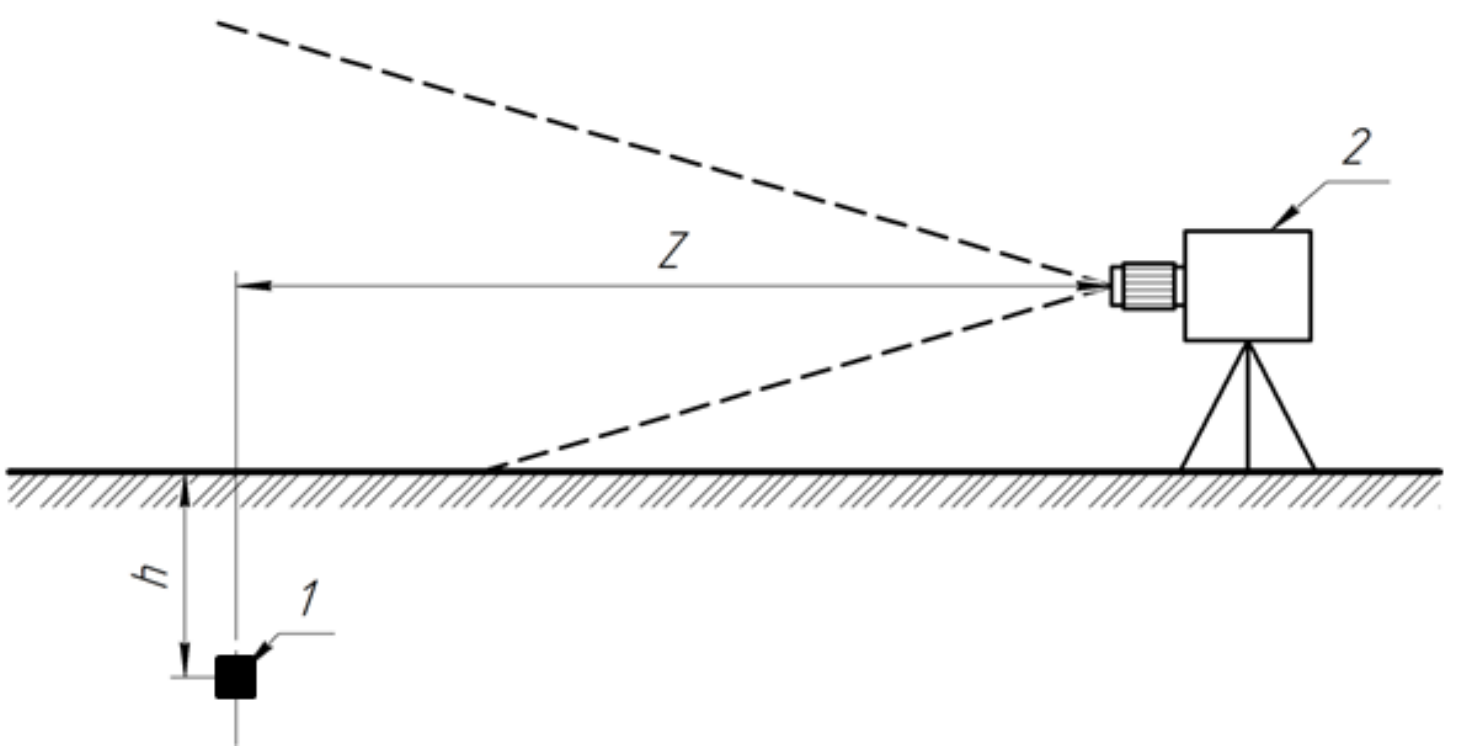

Figure 1-video recording scheme: 1-explosives charge (HE), 2-high-speed video camera. 


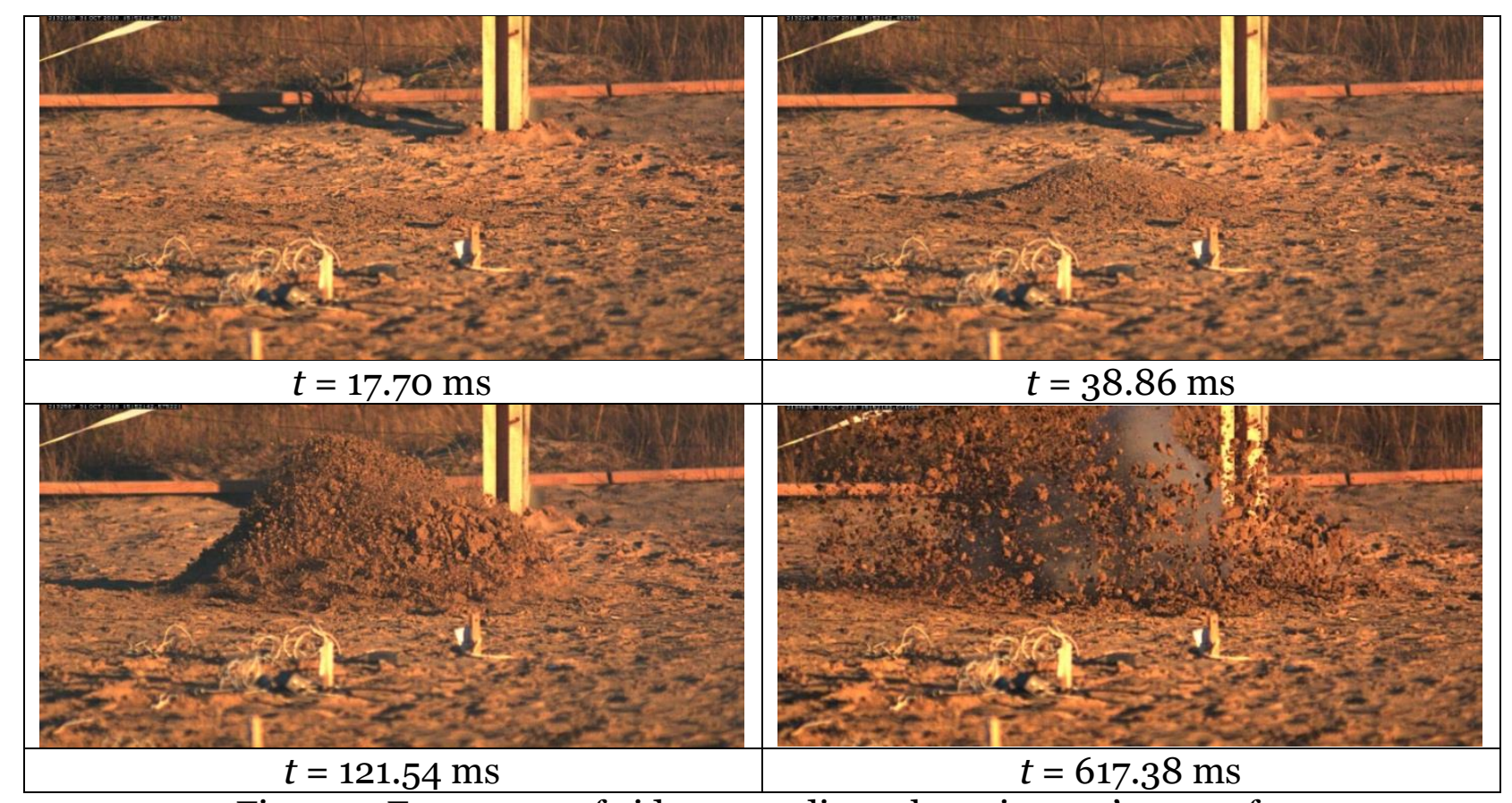

Figure 2-Fragments of video recording the epicenter's area of a deep explosive charge explosion.

\section{Numerical simulation}

Numerical simulation was performed using an explicit solver on a three-dimensional Euler grid.

The system of equations describing the flow of the medium [7] has the form

$$
\left\{\begin{array}{l}
\frac{\partial \rho}{\partial t}+\nabla \rho(\boldsymbol{v})+\rho \operatorname{div} v=0 \\
\rho \frac{\partial \boldsymbol{v}}{\partial t}+\rho \nabla \boldsymbol{v}(\boldsymbol{v})=\operatorname{div} \sigma \\
\rho \frac{\partial E}{\partial t}+\rho \nabla E(\boldsymbol{v})=\sigma \cdot \dot{\varepsilon}
\end{array}\right.
$$

where $\rho$ - the density of medium, $\mathbf{v}$ - the velocity vector of medium, $\mathbf{F}$ - the vector field of mass forces,

$\sigma$ - stress tensor, $\varepsilon$ - strain rate tensor, $E$ - internal energy $t$ - current time.

The stress-strain state at a point in the calculated area was determined by the General system of equations:

$$
\begin{gathered}
\sigma_{i j}=-p \cdot \delta_{i j}+s_{i j} \\
p=p(\rho, E),
\end{gathered}
$$

where $\sigma_{i j}$-stress tensor, $s_{i j}$ - stress tensor deviator, $\delta_{i j}$ - Kronecker's symbol, $p$ - the hydrostatic pressure given by the equation of state (SE).

The geometric parameters of the calculation model are shown in figure 3. Each part of the calculation model corresponds to its own mathematical model of the material. 


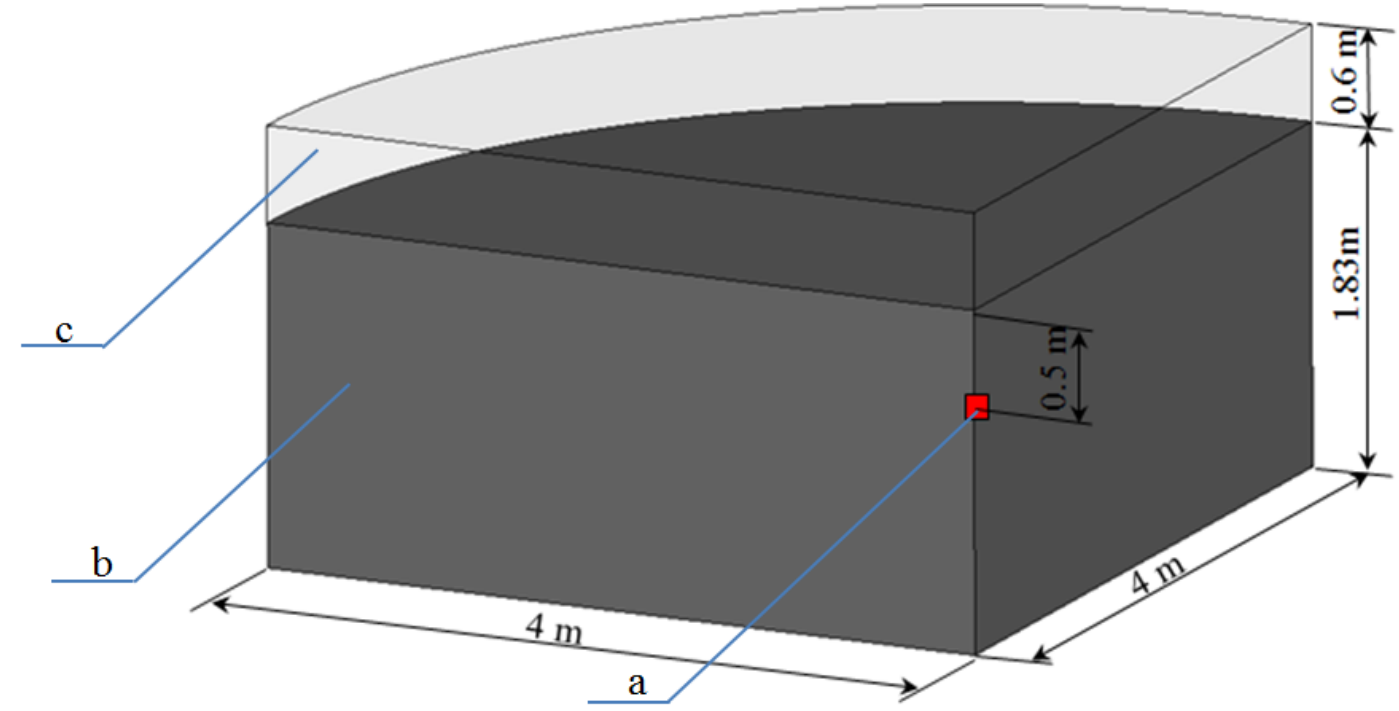

Figure 3-Geometry of the calculation model: a-explosive substance, b-sandy ground, c-air.

Air in the calculation was described as a continuous medium with the equation of state of an ideal gas:

$$
p=(\kappa-1) \frac{\rho}{\rho_{0}} E
$$

where $k=1,4$ - adiabatic index, $E$-internal energy per unit volume, $\rho_{0}$ - initial density.

To specify the explosive material, a special model of explosive was used, which allows modeling the detonation and flow of explosion products $[8,9]$. The pressure in the element $\mathrm{HE}$ in each moment of time is determined by the formula

$$
p=F \cdot p(\rho, E) \text {, }
$$

where $F=\max \left(F_{1}, F_{2}\right)$ - percentage of explosive burnout $F_{1}=\frac{\rho \cdot D^{2}}{P_{\mathrm{CJ}}}\left(1-\frac{V}{V_{0}}\right), F_{2}=\frac{2 D\left(t-t_{b}\right)}{3 \Delta x}$, $\rho$ - density explosives, $D$ - detonation velocity, $P_{C J}$ - pressure Chapman-Jouguet, $t_{b}$ - element burn-out time, $\Delta x$ - the characteristic element size.

SE for explosives detonation products is accepted in the form of Jones-Wilkins-Lee [8, 10]:

$$
p(\rho, E)=A \cdot\left(1-\frac{\varpi}{R_{1} \cdot V}\right) e^{-R_{1} V}+B\left(1-\frac{\varpi}{R_{1} \cdot V}\right) e^{-R_{2} V}+\frac{\varpi \cdot E}{V},
$$

where $V=\frac{\rho_{0}}{\rho}$ is the relative volume of detonation products HE during the explosion, $A, B$, $R 1, R 2, \omega$ are empirical constants, $E$ is the internal energy assigned to a unit of volume, $\varpi=-\left(\frac{\partial \ln p}{\partial \ln V}\right)_{S}-1$ - when the explosion products expand to a value of $V>1 O, S$ is the entropy.

Equation (1) corresponds to the isentrope of the explosion products:

$$
p_{s}=A \cdot e^{-R_{1} V}+B e^{-R_{2} V}+C \cdot V^{-(1+\varpi)}
$$

The parameters of the HE material model adopted in accordance with [11] are shown in table.1.

The parameters of SE HE are taken in accordance with [11] and are shown in table. 2.

Table 1 - Parameters of the explosives material model

\begin{tabular}{|c|c|c|}
\hline $\begin{array}{c}\text { Explosives density } \\
\rho, \mathrm{kg} / \mathrm{m}^{3}\end{array}$ & Detonation speed $\mathrm{D}, \mathrm{km} / \mathrm{s}$ & $\begin{array}{c}\text { Pressure Chapman-Jouguet } \\
P_{C J}, \mathrm{GPa}\end{array}$ \\
\hline 1821 & 8.48 & 34.2 \\
\hline
\end{tabular}


Table 2-Parameters of the equation of state of detonation product HE

\begin{tabular}{|c|c|c|c|c|}
\hline$A, G P a$ & $B, G P a$ & $R_{1}$ & $R_{2}$ & $\omega$ \\
\hline 748.6 & 13.38 & 4.5 & 1.2 & 0.38 \\
\hline
\end{tabular}

Elastic properties of sandy ground were assumed according to Table 3 [12].

Table 3-Elastic properties of sand

\begin{tabular}{|c|c|c|}
\hline $\begin{array}{c}\text { Density of sand } \\
\rho_{0}, \mathrm{~g} / \mathrm{cm}^{3}\end{array}$ & $\begin{array}{c}\text { Shear modulus } \\
G, M P a\end{array}$ & Poisson Ratio $v$ \\
\hline 1.5 & 150 & 0.3 \\
\hline
\end{tabular}

The dependence of the yield strength of sand on pressure was determined by the ratio in accordance with [13]

$$
Y=Y_{0}+\frac{\mu P}{1+\frac{\mu P}{\left(Y_{1}-Y_{0}\right)}}
$$

where $Y_{0}$ is the adhesion (strength at zero pressure)

$Y_{1}$ - the value that restricts the intensity of shear stresses from above:

$Y_{1}=50 \exp \left(\frac{-20.7 W}{1+W}\right) M \Pi a[13], \mathrm{W}-$ weight humidity in fractions of a unit.

$\mu=2 \cdot \operatorname{tg} \varphi-$ the value associated with the coefficient of internal friction, $\varphi-$ the angle of internal friction.

Parameter values, and are shown in Table. 4.

Table 4 - Parameters of sandy ground taken in the calculation [12]

\begin{tabular}{|c|c|c|c|}
\hline $\begin{array}{c}\text { Density of sand } \\
\rho_{0}, g / \mathrm{sm}^{3}\end{array}$ & $Y_{0}, M P a$ & $Y_{1}, M P a$ & $\mu$ \\
\hline 1.5 & 0.01 & 8 & 0.91 \\
\hline
\end{tabular}

A graphical representation of the dependence of the yield strength on pressure is shown in Figure 4 .

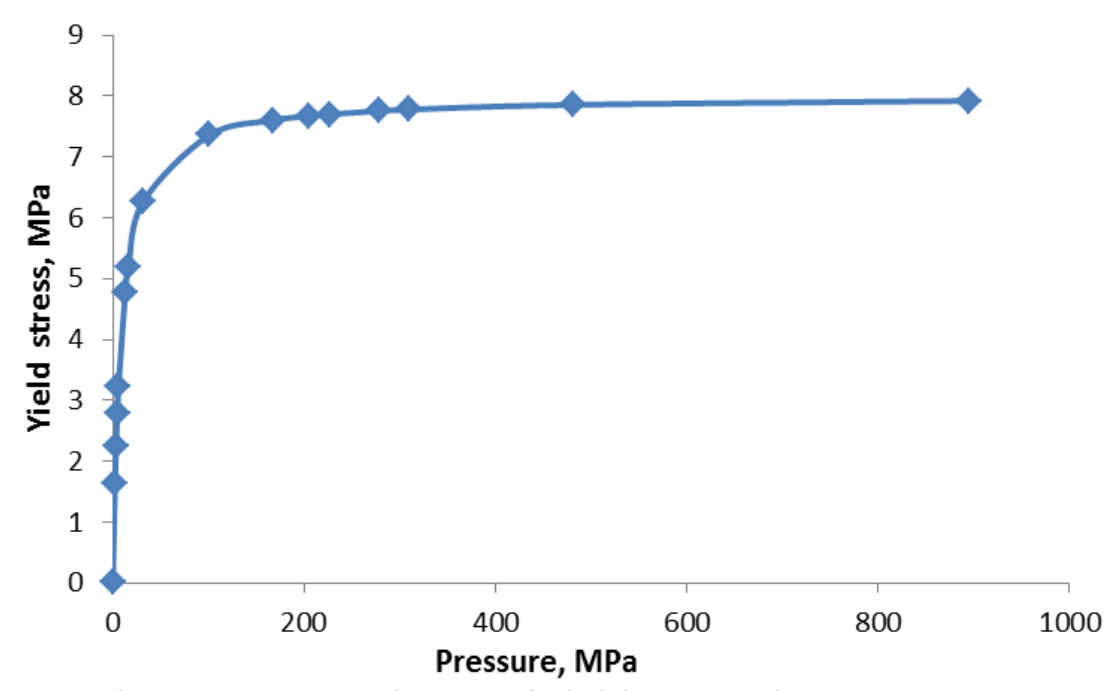

Figure 4-Dependence of yield strength on pressure 
The graph of the logarithm's dependence of the relative volume on the pressure when compressing sandy ground is shown in Figure 5 .

On the graph, $V / V 0=1-\theta$, where $\mathrm{V}$ - the current volume, Vo - the initial volume, and $\theta$ the volumetric strain. The dependence of the volume strain on the pressure has the form [14]:

$$
P=\frac{\rho_{0} \cdot A^{2} \theta}{\left(1+\frac{4 \operatorname{tg} \varphi}{3}\right) \cdot(1-B \cdot \theta)^{2}}
$$

where $\operatorname{tg} \varphi=0.6$ - the internal friction angle tangent, $A=511 \mathrm{~m} / \mathrm{s}$ is the coefficient of impact adiabate [12], $\mathrm{B}=1.72$ is the coefficient of impact adiabate [12].

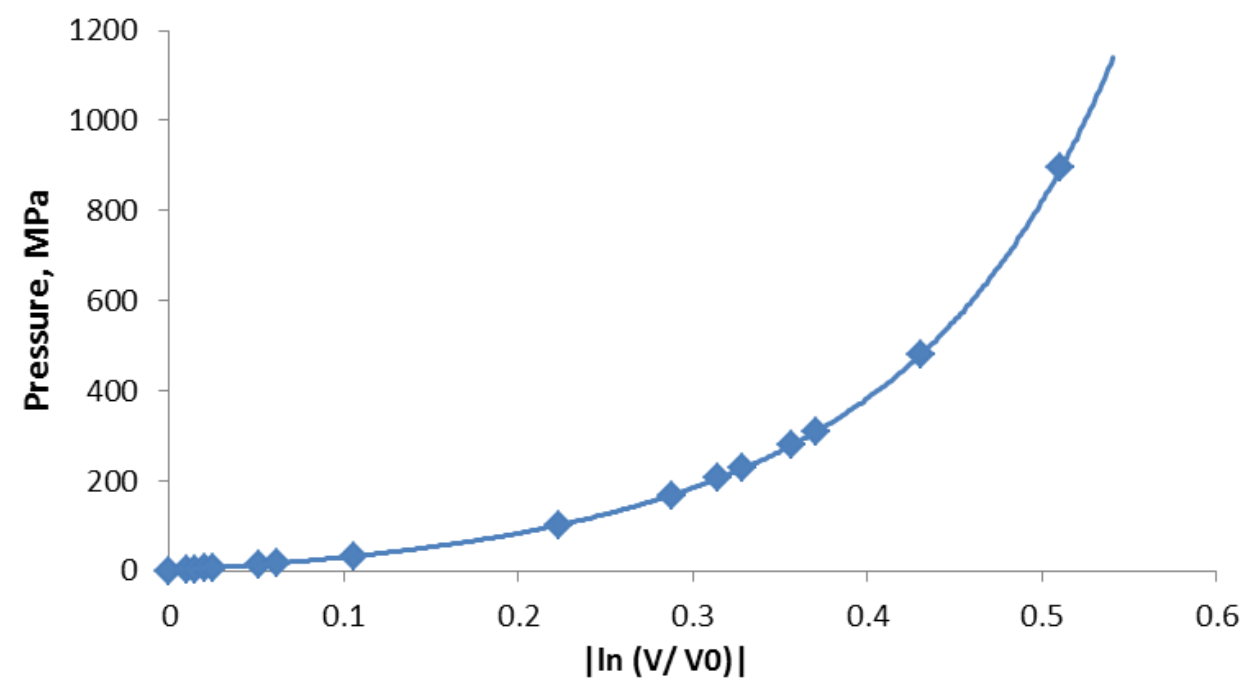

Figure 5-Dependence of strain on pressure

The Unloading module for different density values $\mathrm{p}$ is defined by the formula [14]

$$
\mathrm{K}=\rho \frac{d f(\rho)}{d \rho}
$$

where $f(\rho)=p$ - the function of density dependence on the pressure.

Numerical simulations were performed using an explicit solver on a three-dimensional Euler grid

\section{Analysis of the results obtained}

The video information obtained in the experiments was used to determine the dome's height of the sandy ground $(\mathrm{H})$ at discrete moments of time. Direct measurement of the dome height by video recording frames caused difficulties, especially for small $\mathrm{H}$ values, related to identifying the top point of the ground dome. To solve this problem, we used cross-correlation processing of video recording frames. A multi pass cross correlation processing algorithm with a square survey window and 50\% overlap was used. The size of the survey window was iteratively reduced from 256 to 8 pixels. Approximation of the correlation function was carried out using three-point Gaussian interpolation without pre-processing images. The fast Fourier transform algorithm was used to calculate the correlation function.

Fragments of the results of cross-correlation processing of video recording frames are shown in Figure 6. The sequential image analysis was performed after the explosion of explosive charge HE with the image of an undisturbed surface of sandy ground. This approach allows to visualize the field areas of displacement of the ground surface where there is no significant change in its structure. The received information can be used for estimating the diameter of the disturbance area (Figure 7), but is not suitable for determining the height of the ground dome. For information about the height of the dome of the sandy ground, the surface structure of which changes, two consecutive images of the sandy surface were analyzed, and the 
maximum change in the analyzed area in the vertical direction was determined (Figure 8). Thus, the increment of the height of the ground dome $\mathrm{H}$ was determined for the time equal to the video registration period The cross-correlation processing algorithm allows to analyze images in the sub-pixel area, in this case, the standard deviation from the increment $\mathrm{H}$ was 0.06 pixels, which, taking into account the registration scheme, is $0.1 \mathrm{~mm}$. Experimental data in comparison with the results of numerical simulation are presented in Figure 9 as a graph of the ground dome height dependence on time

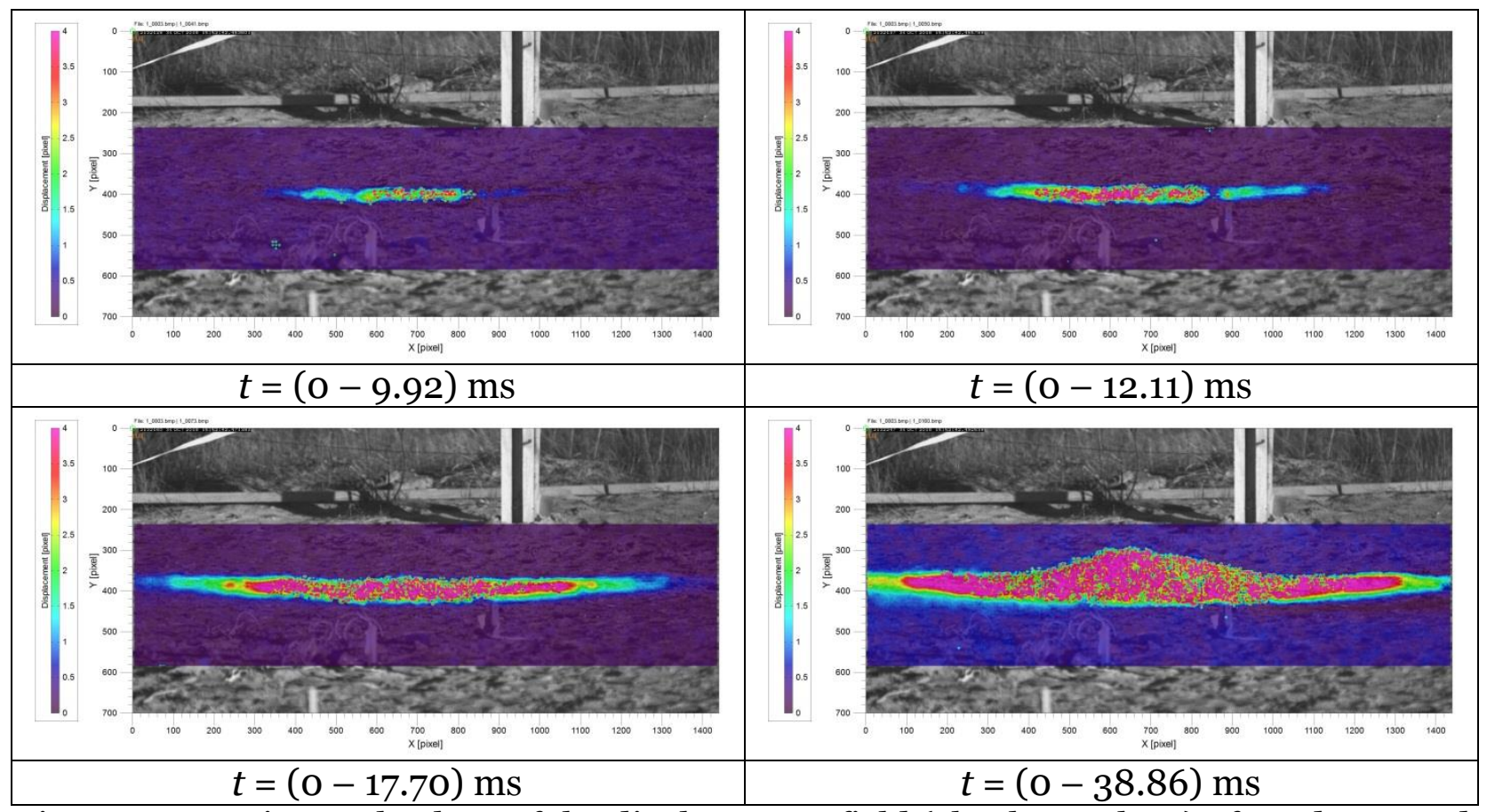

Figure 6-Experimental values of the displacement field (absolute values) of sandy ground relative to its initial (undeformed) state

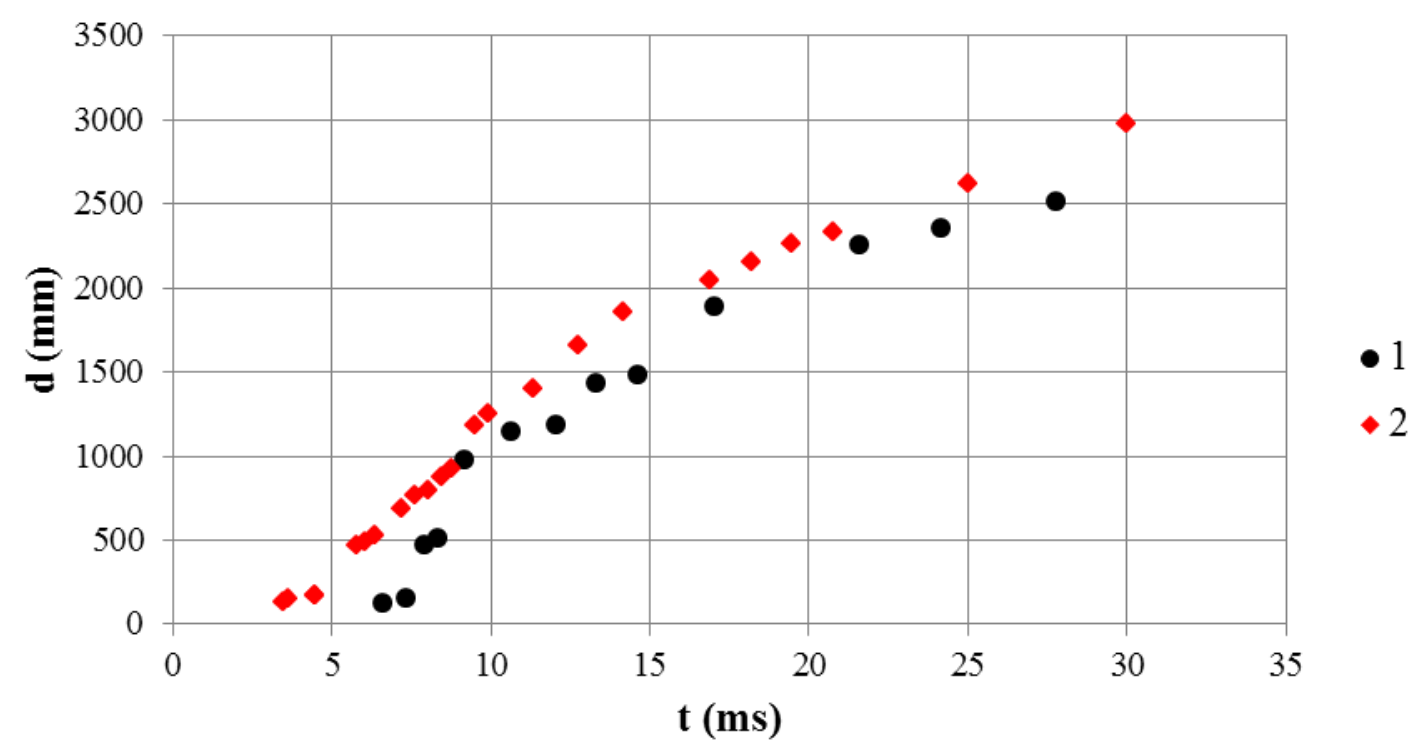

Figure 7-Estimated data on the diameter of the perturbation area: 1 - experimental data at $\mathrm{h}=0.55 \mathrm{~m}, 2$-experimental data at $\mathrm{h}=0.5 \mathrm{~m}$. 


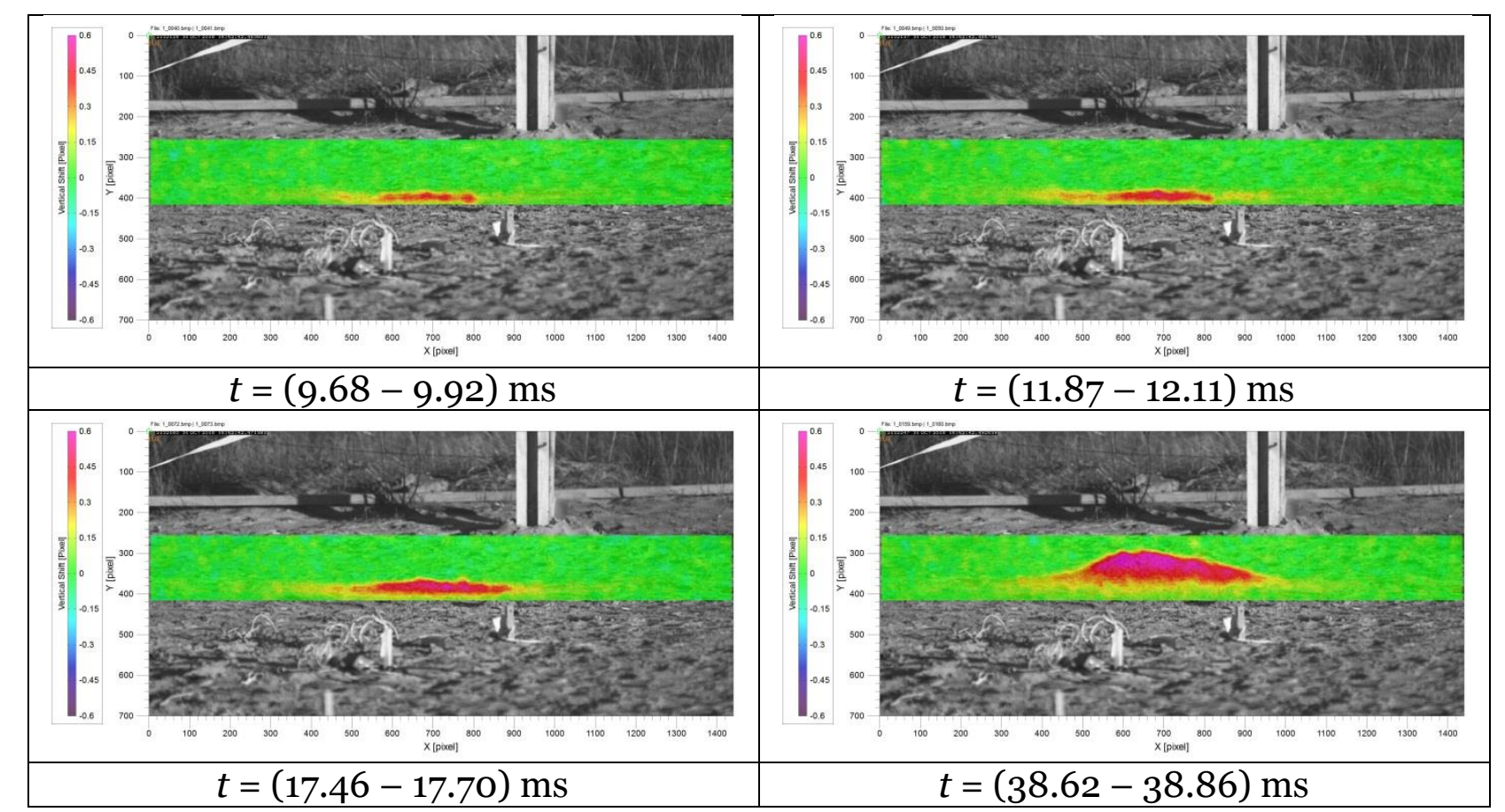

Figure 8-Experimental values of the displacement field (vertical component) of sandy ground that occurred during the frame period of video registration

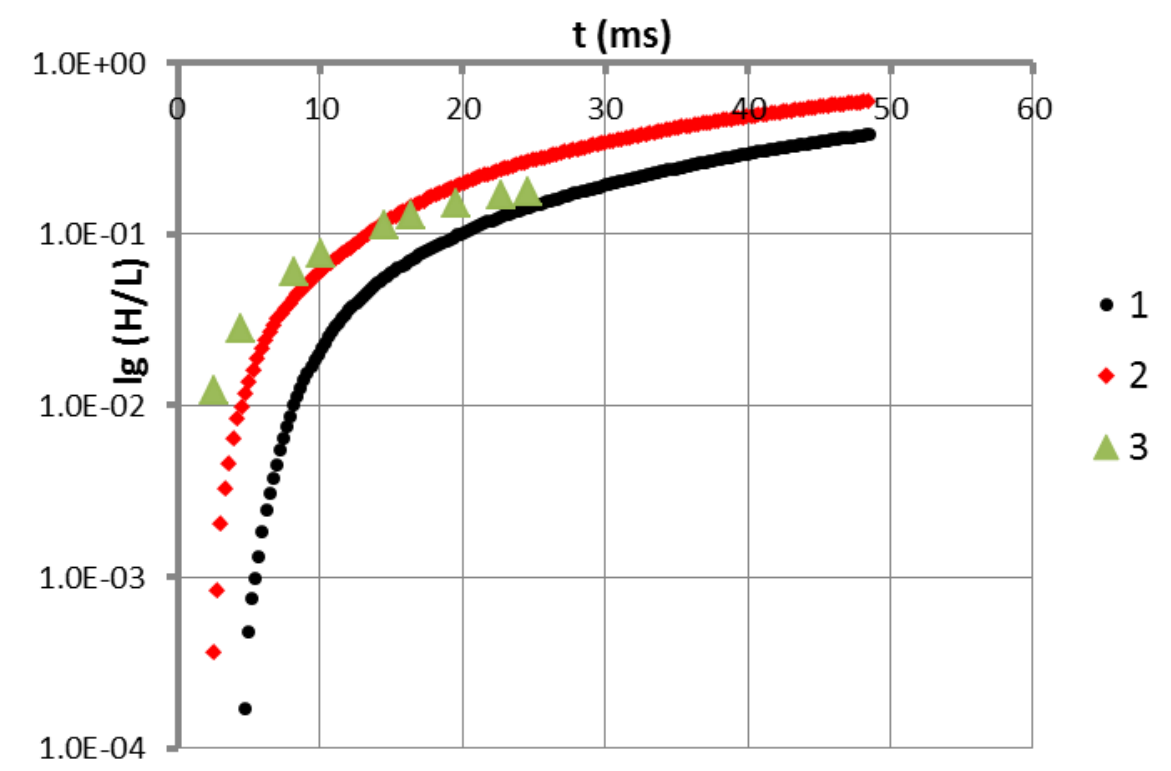

Figure 9 - Dependence of the height of the ground dome on time: 1-experimental data at $\mathrm{h}=0.55 \mathrm{~m}$, 2-experimental data at $\mathrm{h}=0.5 \mathrm{~m} ; 3$-calculated data for $\mathrm{h}=0.5 \mathrm{~m}$.

From the analysis of the results of experiments and calculations, it can be concluded that the ground dome's rate of growth increases up to a certain point in time, after which it becomes almost constant. This can probably be explained by the fact that at the initial stage of the explosion's development in the cavern, the high pressure of the explosion products promotes accelerated movement of a solid mass of ground. After the expansion of the cavern, the pressure of the explosion products decreases - the rate of growth of the dome height also decreases.

The comparison of calculated and experimental data is given up to the time of $25 \mathrm{~ms}$, since after that the dome begins to fragment into separate parts, which cannot be described by numerical algorithms within the continuum model. 


\section{Conclusion}

The paper considers an experimental and computational investigation of a cylindrical charge explosion in dry sandy ground with a density of $1.5 \mathrm{~g} / \mathrm{cm}^{3}$. During the experiment, optical and physical registration of the disturbance development process on the ground surface was used. The diameters of the perturbation zone and the height of the dome were determined using a cross-correlation image algorithm. The correlation function was approximated using threepoint Gaussian interpolation without pre-processing images. The fast Fourier transform algorithm was used to calculate the correlation function. The results of numerical simulation are also presented. The comparison of the calculated data on the height of the sandy ground discharge and the experimental data on the height comparison showed their satisfactory correspondence, which indicates the adequacy of the applied mathematical model.

The work was supported by the grant of the President of the Russian Federation no. MK2078.2019.8.

\section{References}

1. Raffel, M. Background-oriented schlieren (BOS) techniques. // Experiments in Fluids. 2015. Vol.56. № 3. P. 1-17.

2. Adrian, R.J. Twenty years of particle image velocimetry. // Experiments in Fluids. 2005. Vol. 39. No. 2. P. 159-169.

3. Interaction of numerical and experimental visualization of flows. I. A. Znamenskaya [et al.] // Scientific visualization. 2013. Vol. 5. No. 3. P. 40-51.

4. Using the shadow background method to study a non-stationary flow with a shock wave. F. N. Glazyrin et al. // Scientific visualization. 2013. Vol. 5. No. 3. P. 65-74.

5. In situ velocity and stress characterization of a projectile penetrating a sand target: experimental measurements and continuum simulations. J.P. Borg et al. // International Journal of Impact Engineering. 2013. Vol. 51. P. 23-35.

6. Computational and experimental study of shock-wave loading of optically transparent bodies. S. I. Gerasimov et al. // Technical Physics. 2019. Vol. 89. No. 9. P. 1319-1324.

7. Muizemnek A.Yu., Bogach A.A. Mathematical modeling of impact and explosion processes in the LS-DYNA program. Penza: Information and publishing center of PSU, 2005.

8. Hallquist J.O. LS-DYNA: Theoretical manual. Livermore Software Technology Corporation, Livermore, 1998. $498 \mathrm{p}$.

9. Souli M. "LS-Dyna Advanced Course in ALE and Fluid/Structural Coupling". Course Note for Arbitrary Lagrangian-Eulerian Formulation Technique. Livermore, LSTC, CA, 2000.

10. Lee E., Finger M., Collins W. JWL equation of state coefficients for high explosives. Rept-UCID-16189, Lawrence Livermore National Laboratory, 1973.

11. Orlenko A.P. Physics of explosion/ edited by L. P. Orlenko. - Ed. 3rd, ISPR. - In 2 t. T. 1. Moscow: FIZMATLIT, 2004.

12. Balandin V.V., Bragov A.M., Igumnov L.A., Konstantinov A.Yu., Kotov V.L., Lomunov A.K. Dynamic deformation of soft ground media: experimental research and mathematical modeling. Solid state mechanics, no. 3, 2015. PP. 69-77.

13. Zamyshlyaev B.V., Evterev L.S. Models of dynamic deformation and destruction of ground media. Moscow: Nauka, 1990. 215 p.

14. Bazhenov V.G., Kotov V.L. Mathematical modeling of non-stationary processes of impact and penetration of axisymmetric bodies and identification of properties of ground media. M: FIZMATLIT, 2011. 208 p. 